\title{
Correction to: Graphical Representation and Similarity Analysis of DNA Sequences Based on Trigonometric Functions
}

\author{
Guo-Sen Xie ${ }^{1,2}$ (D) Xiao-Bo Jin ${ }^{3} \cdot$ Chunlei Yang ${ }^{1,2} \cdot$ Jiexin Pu ${ }^{1,2} \cdot$ Zhongxi Mo $^{4}$
}

Published online: 5 June 2018

(C) Springer Science+Business Media B.V., part of Springer Nature 2018

\section{Correction to: Acta Biotheor (2018) 66:113-133 https://doi.org/10.1007/s10441-018-9324-0}

In the original publication of the article, the y axis labels present in Figs. 1a and 2a are incorrect. The correct Figs. 1a and 2a are provided here.

The original article can be found online at https://doi.org/10.1007/s10441-018-9324-0.

\section{Guo-Sen Xie}

gsxiehm@gmail.com

1 Information Engineering College, Henan University of Science and Technology, Luoyang 471023, China

2 Henan Joint International Research Laboratory of Image Processing and Intelligent Detection, Henan University of Science and Technology, Luoyang 471023, China

3 School of Information Science and Engineering, Henan University of Technology, Zhengzhou 450001, China

4 School of Mathematics and Statistics, Wuhan University, Wuhan 430072, China 
Fig. 1 a The assignment of the sequence $\mathrm{S}=\mathrm{ATGGTG}$ CACCTGACTCCTGA to four sinusoidal functions. b Four-Base-related curve of the sequence S. c A-related curve, G-related curve, T-related curve and $\mathrm{C}$-related curve of the sequence $\mathrm{S}$ (a)

A $8 \longdiv { \text { Assign Bases to Sinusoidal Functions } }$

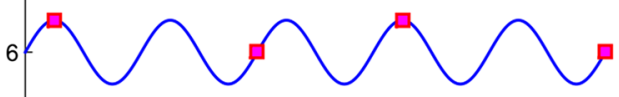

G

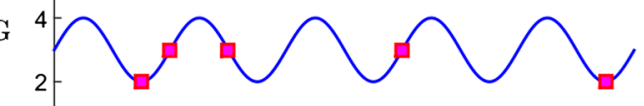

$\mathrm{T}$

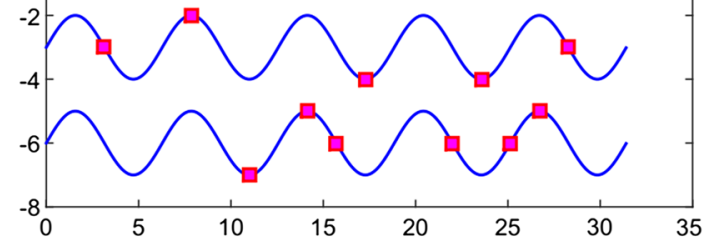

(b)

Sinusoidal Four-Base Related Curve

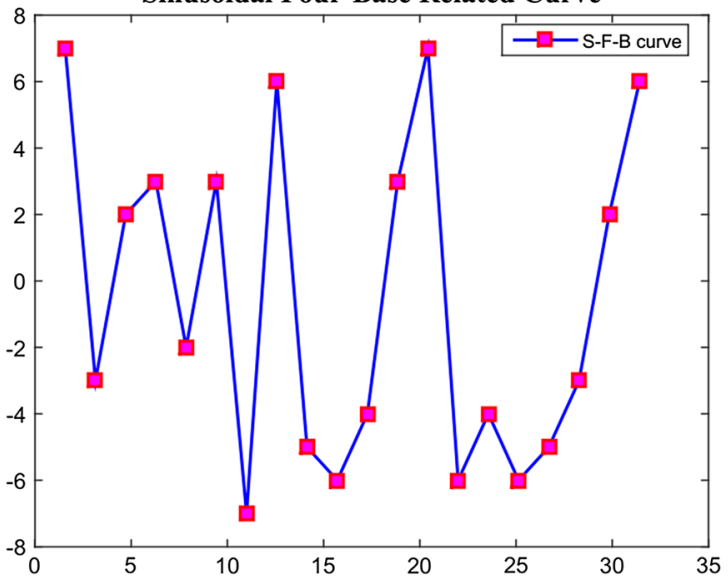

(c) Sinusoidal Single-Base Related Curves

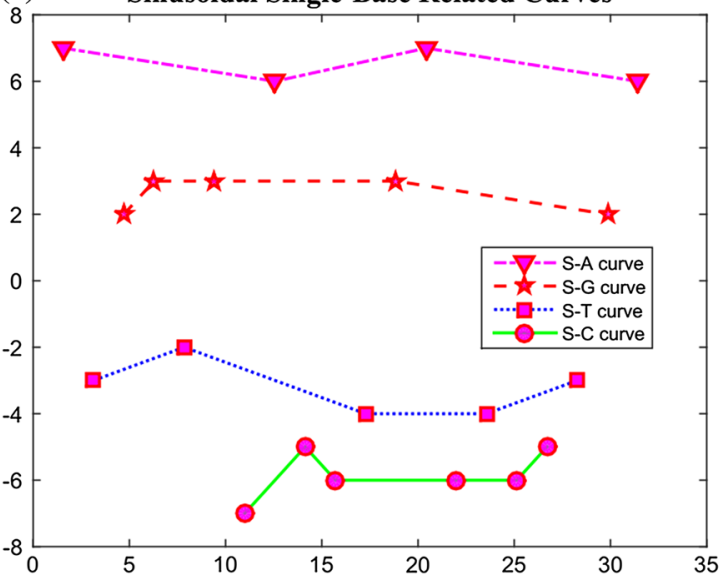


Fig. 2 a The assignment of the sequence $\mathrm{S}=\mathrm{ATGGTG}$ CACCTGACTCCTGA to four tangent functions. b Four-Baserelated curve of the sequence $S$. $c$ A-related curve, G-related curve, T-related curve and C-related curve of the sequence $\mathrm{S}$

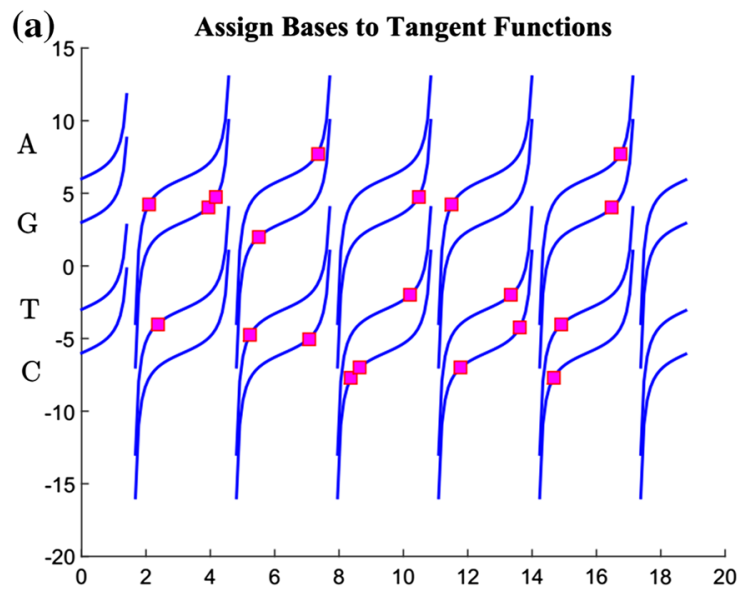

(b)

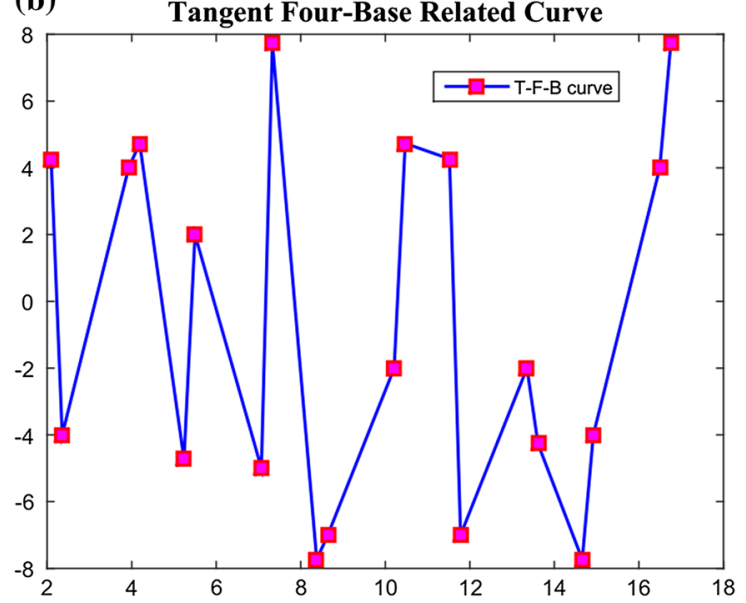

(c) Tangent Single-Base Related Curves

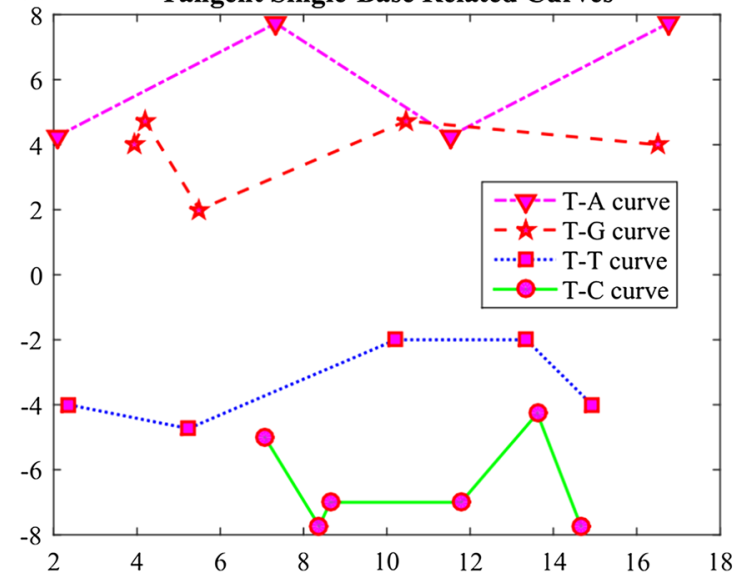

